\title{
Is Epicardial Adipose Tissue Associated with Atrial Fibrillation Following Cardiac Surgery? A Systematic Review and Meta-Analysis
}

\author{
Rina Sha, MD,${ }^{1}$ Wenqiang Han, MD, ${ }^{1}$ Mingjie Lin, $\mathrm{PhD},{ }^{1,2}$ Jingquan Zhong, $\mathrm{PhD},{ }^{1,3}$ \\ ${ }^{1}$ The Key Laboratory of Cardiovascular Remodeling and Function Research, Chinese Ministry of Education, Chinese National Health \\ Commission and Chinese Academy of Medical Sciences, The State and Shandong Province Joint Key Laboratory of Translational \\ Cardiovascular Medicine, Department of Cardiology, Qilu Hospital, Cheeloo College of Medicine, Shandong University, Jinan, China; \\ ${ }^{2}$ Peking University First Hospital, Beijing, China; ${ }^{3}$ Department of Cardiology, Qilu Hospital (Qingdao), Cheeloo College of Medicine, \\ Shandong University, Qingdao, China
}

\section{ABSTRACT}

Background: Although epicardial adipose tissue (EAT) has been proven to be related to atrial fibrillation (AF) and post-ablation AF recurrence, the association between EAT and $\mathrm{AF}$ after cardiac surgery (AFACS) remains unclear.

Objective: This study was a systematic review and meta-analysis that assessed the relationship between EAT and AFACS.

Methods: Electronic databases were systematically searched for "atrial fibrillation" and "epicardial adipose tissue." The analysis was stratified according to the EAT measurement into three meta-analyses as (1) total EAT volume, (2) left atrial (LA)-EAT volume, and (3) EAT thickness. Standardized mean difference (SMD) was estimated using a random effects model.

Results: Eight articles with 10 studies (546 patients) were included. The meta-analysis revealed that EAT was higher in those with AFACS irrespective of the EAT measurement (total EAT volume: $\mathrm{SMD}=0.56 \mathrm{~mL}, 95 \%$ confidence interval, $\mathrm{CI}=0.56-1.10 \mathrm{~mL}, \mathrm{I}^{2}=0.90, P=.04$; EAT thickness: $\mathrm{SMD}=0.85 \mathrm{~mm}, 95 \% \mathrm{CI}=0.04-1.65 \mathrm{~mm}, \mathrm{I}^{2}=0.90, P=.04$; LA-EAT volume: $\mathrm{SMD}=0.57 \mathrm{~mL}, 95 \% \mathrm{CI}=0.23-0.92 \mathrm{~mL}$, $\left.\mathrm{I}^{2}=0.00, P=.001\right)$.

Conclusion: EAT was higher in patients with AFACS, measured either as volume or thickness.

\section{INTRODUCTION}

\section{Background}

Atrial fibrillation (AF) is the one of the most common arrhythmia with an increasing rate of morbidity and mortality [Gaita 2018]. AF after cardiac surgery (AFACS) is one of the most common postoperative complications of cardiac surgical procedures; it occurs in up to $30 \%$ of patients

Received May 14, 2021; received in revised form fuly 24, 2021; accepted fuly 26, 2021.

Correspondence: Fingquan Zhong, Department of Cardiology, Qilu Hospital of Shandong University, No. 107 Wenhuaxi Road, Finan 250012, China; +8618560086597; fax: 86-531-86927544 (e-mail: 18560086597@163.com).
[D'Agostino 2018]. Although cardiac surgery-associated morbidity and mortality have reduced, the incidence of AFACS remains almost unchanged [Shen 2011; D'Agostino 2016]. AFACS may have a negative influence on postoperative results in the long term. It can result in a two-fold increase in all-cause postoperative 30-day and 6-month mortality compared with those in patients with sinus rhythm [Schwann 2018].

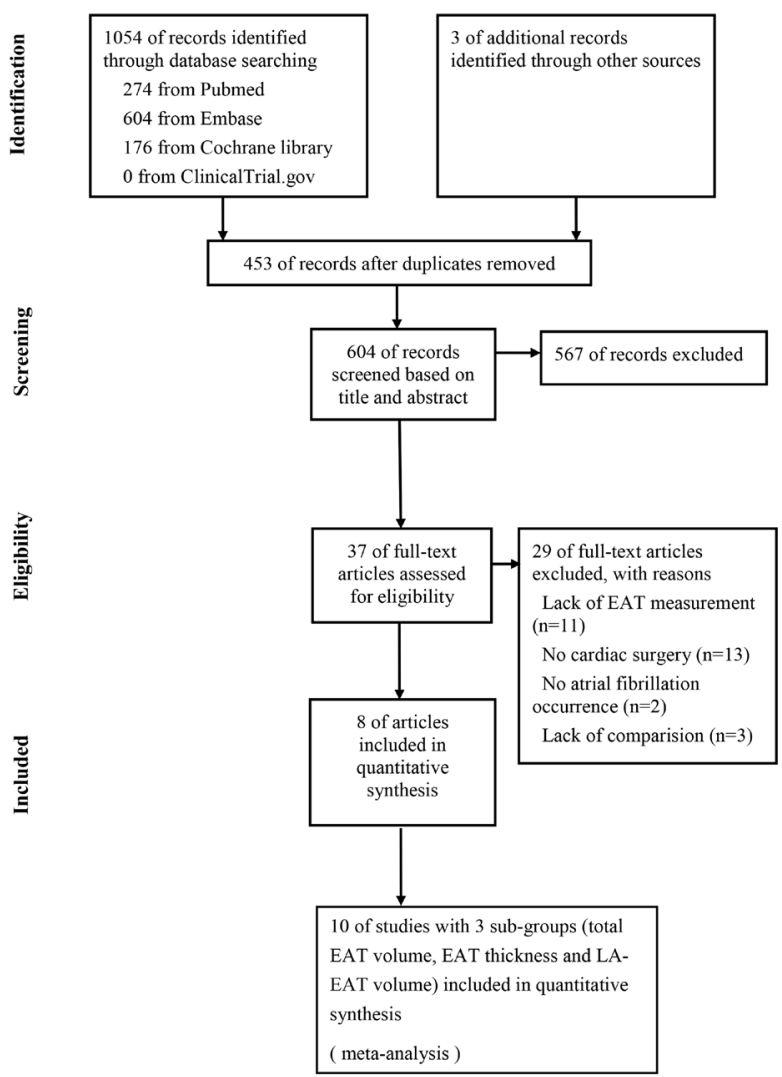

Figure 1. Flow chart of the articles included in the study. EAT, epicardial adipose tissue 
Table 1. Characteristics of the included studies

\begin{tabular}{|c|c|c|c|c|c|c|c|c|c|}
\hline Drossos & 2014 & Greece & 83 & $28,195 \pm 80$ & $55,126 \pm 47$ & EAT volume $(\mathrm{ml})$ & CT & OA & $19 / 22$ \\
\hline Ozbek & 2018 & Turkey & 149 & $35,135.71 \pm 46.78$ & $114,118.71 \pm 42.85$ & EAT volume (ml) & CT & CA & $4 / 12$ \\
\hline Rachwalik & 2019 & Poland & 46 & $14,7.1 \pm 1.2$ & $32,7.4 \pm 1.2$ & EAT thickness (mm) & Echo & $O A$ & $18 / 22$ \\
\hline Wang (VHD) & 2018 & China & 49 & $20,7.62 \pm 0.69$ & $57,7.14 \pm 1.14$ & EAT thickness (mm) & Echo & OA & $17 / 22$ \\
\hline Liu & 2019 & China & 74 & $37,5.6 \pm 1.1$ & $37,5 \pm 1.3$ & EAT thickness (mm) & Echo & OA & $20 / 22$ \\
\hline Gunturk & 2020 & Turkey & 125 & $45,7.28 \pm 0.57$ & $80,6.42 \pm 0.35$ & EAT thickness $(\mathrm{mm})$ & Echo & OA & $19 / 22$ \\
\hline Opolski & 2015 & Poland & 102 & $24,5.6 \pm 3.0$ & $78,4.0 \pm 2.5$ & LA EAT volume $(\mathrm{ml})$ & CT & OA & $19 / 22$ \\
\hline Kogo (total EAT) & 2018 & Japan & 77 & $21,177.7 \pm 69.8$ & $56,165.9 \pm 65.9$ & EAT volume (ml) & CT & OA & $18 / 22$ \\
\hline
\end{tabular}

AF, atrial fibrillation; EAT, epicardial adipose tissue; SD, standard deviation; CT, computed tomography; OA, original article; CA, conference abstract; VHD, valvular heart disease; NVHD, non-valvular heart disease; LA, left atrium. (Note: Unit of the volume and thickness variables are milliliter (ml) and millimeter $(\mathrm{mm})$, respectively)

Recently, more and more people have begun to pay attention to the association between epicardial adipose tissue (EAT) and AF because of its inflammatory and endocrine properties [Wong 2017]. EAT is an ectopic adipose tissue, including fat depots (such as pericardial fat, perivascular fat, and myocardial steatosis), between the myocardium and visceral pericardium. Based on the location, it is easy to understand the physiological functions of the EAT, which include supplying the cardiomyocytes with energy from lipids storage, protecting autonomic ganglia and nerve tissue, and regulating coronary vessels [Rachwalik 2019]. Therefore, EAT may play a central role in cardiovascular disease pathogenesis [Zhou 2020]. Several studies have demonstrated an increasing correlation between EAT and coronary artery disease, atherosclerosis, and progression of coronary plaque burden [Rosito 2008; Ding 2009; Mahabadi 2009], major adverse cardiovascular events [Cheng 2010], and AF [Venteclef 2015; Thanassoulis 2010; Wong 2011; Batal 2010]. These findings suggested that EAT has potential to be a biomarker for cardiovascular disease in clinical application.

The relationship between EAT and AFACS first was described by Drossos et al [Drossos 2014] in 2014. Since then, more than 10 studies have been performed on this topic. However, they have been done in different populations and included different procedures (coronary artery bypass grafting or valve replacement) with different EAT measurements (computed tomography [CT] and echocardiography) and diverse intervals of follow up [Rachwalik 2019; Drossos 2014; Wang 2019; Opolski 2015; Kogo 2019; Liu 2020; Gunturk 2020; Ozbek 2018]. This study is the first attempt to prove the association between AFACS and EAT in patients with and without AF following cardiac surgery, via systematic review, and meta-analysis.

\section{MATERIALS AND METHODS}

\section{Search strategy}

The search was performed using the PubMed, Cochrane, and Embase databases with the note before May 30, 2020. The search was performed using the following expressions: [('epicardial adipose tissue' OR 'epicardial fat' OR 'pericardial adipose tissue' OR 'pericardial fat' OR 'paracardial adipose tissue' OR 'paracardial fat') AND ['atrial fibrillation (MeSH Terms)'] to discern and search all potentially relevant researches. Additionally, manual searches of the reference list of the included studies were performed. We conducted this meta-analysis following the Preferred Reporting Items for Systematic Reviews and Meta-Analyses guidelines. The systematic review was registered in PROSPERO (ID: 219400). As per the different measuring methods of EAT, the eligible studies were categorized into three sub-groups of total EAT volume, EAT thickness, and LA-EAT volume.

\section{Eligibility criteria}

Case reports and review articles were excluded. Only English publications in peer-reviewed journals were eligible for this meta-analysis if they: (1) reported EAT thickness or volume with at least one of the following main confounders: age, sex, hypertension, and body mass index (BMI); (2) measured EAT indexes in two groups of patients-those with AFACS and without AFACS; and 3) evaluated EAT using CT, magnetic resonance imaging, or echocardiography. The literature search was independently evaluated by two specialists to assure conformity to the article's inclusion criteria. If no agreement could be reached, it was decided by a third (senior) author.

\section{Study quality assessment}

The Strengthening the Reporting of Observational Studies in Epidemiology (STROBE) Statement [von Elm 


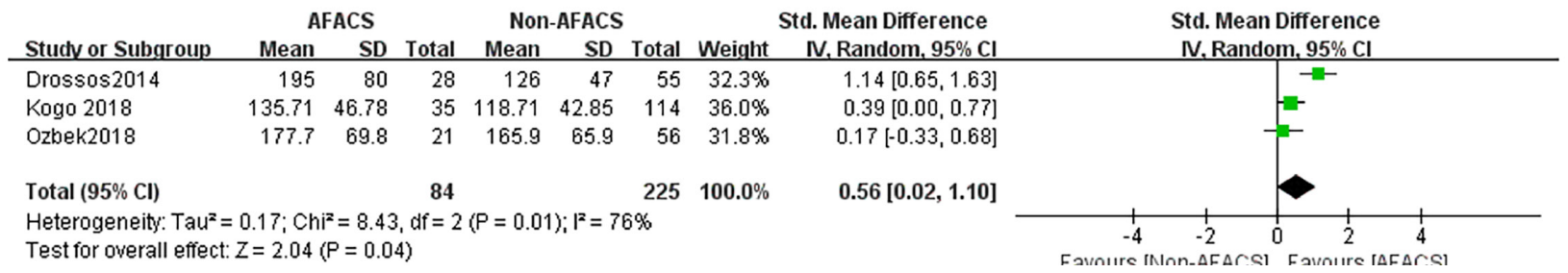

Figure 2. Random effects meta-analysis comparing total EAT volume between patients with and those without AF.

2014] was utilized to evaluate the quality of the included studies. The STROBE guidelines consist of 22 items that assess the quality of the information reported in the abstract, introduction, study design and settings, statistical evaluations, results, discussion, and findings. The included studies were scored on a rating scale of $0-22$, with a higher score reflecting higher quality. The STROBE score was independently evaluated by two investigators. A third senior author was consulted in cases of disagreements. In view of conference abstracts without complete articles available, we used another 12-item STROBE checklist. All scores are summarized in Table 1.

\section{Statistical methods}

The Cochrane $\mathrm{Q}$ test and $\mathrm{I}^{2}$ index were utilized to evaluate individual studies' heterogeneity; $\mathrm{I}^{2}>50 \%$ suggested high heterogeneity. All studies adopted a random effects model in view of high heterogeneity. Considering the small sample size, we used sensitivity analysis to explore the probable sources of heterogeneity. The possibility of publication bias was assessed with funnel plots and Egger's regression test. Statistical analysis was executed using the STATA package v16 (StataCorp, College Station, Texas, USA). $P<.05$ was considered statistically significant.

\section{RESULTS}

\section{Study selection}

The literature search showed 1054 articles, and three more records were identified through a hand search of the reference lists of the selected articles. Overall, 453 duplicated records and 567 topics irrelevant to the subject were excluded. After reading the full text of the 37 remaining articles, 29 articles were excluded because of a lack of EAT measurement $(\mathrm{N}=$ $11)$, cardiac surgery $(\mathrm{N}=13), \mathrm{AF}(\mathrm{N}=2)$, or comparisons $(\mathrm{N}=3)$. Ultimately, the 10 studies published in eight articles [Rachwalik 2019; Drossos 2014; Wang 2019; Opolski 2015; Kogo 2019; Liu 2020; Gunturk 2020; Ozbek 2018] with three sub-groups were included in the study (Figure 1).

\section{Study characteristics}

Patients with $\mathrm{AF}(\mathrm{N}=154)$ and those without $\mathrm{AF}(\mathrm{N}=$ 392) postoperatively were labeled as AFACS and non-AFACS, respectively. Most [Rachwalik 2019; Drossos 2014; Wang 2019; Opolski 2015; Kogo 2019] of the studies included fulltext articles, while only one study [Ozbek 2018] was available as a conference abstract. EAT measurement involved EAT volume and LA-EAT volume, which were assessed using CT and EAT thickness assessed using echocardiography. EAT thickness was defined as an echo-free region between the right ventricular outer wall and the visceral pericardium. Other patient demographics and basic characteristics are summarized in Table 1.

\section{Total epicardial fat volume}

Three studies [Drossos 2014; Kogo 2019; Ozbek 2018] (309 patients) included EAT volume. The comparison of total EAT volume between AFACS and non-AFACS revealed SMD of $0.56 \mathrm{~mL}(95 \%$ confidence interval $[\mathrm{CI}]=0.02-1.10$, $P=.04)$, the result suggesting that EAT volume was higher in AFACS patient group. Apparent significant heterogeneity was found between the studies $\left(\mathrm{I}^{2}=76 \%, P=.01\right)$ (Figure 2A).

\section{Epicardial fat thickness}

Five studies [Rachwalik 2019; Wang 2019; Liu 2020; Gunturk 2020] (334 patients) assessed EAT thickness using echocardiography. Patients with AFACS had a significantly higher EAT thickness than those without AFACS (SMD = $0.85 \mathrm{~mm}, 95 \% \mathrm{CI}=0.04-1.65, P=.04 ; \mathrm{I}^{2}=90 \%$ ) (Figure 2B).

Left atrium epicardial fat volume: Two studies [Opolski 2015; Kogo 2019] (178 patients) measured LA-EAT. Their results demonstrated that there was a significant distinction in SMD between AFACS and non-AFACS $(0.57 \mathrm{~mL}, 95 \% \mathrm{CI}$ $\left.=0.23-0.92, P=.001 ; \mathrm{I}^{2}=0.0 \%\right)$, thus, suggesting a higher LA-EAT volume in patients with AFACS (Figure 2C).

\section{Quality assessment and publication bias}

The quality of the articles in this meta-analysis varied to a great extent. The STROBE scores were 17 to 20 for the full-text articles (Table 1). Potential sources of bias deserved further exploring. Furthermore, the eligibility criteria for patients were not explicit in most research. Figure 3 illustrates the relative symmetry of the funnel plots of publications (Figure 3). However, according to the Egger test for small-study effects, there was no evidence of publication bias $(P=.160)$. 


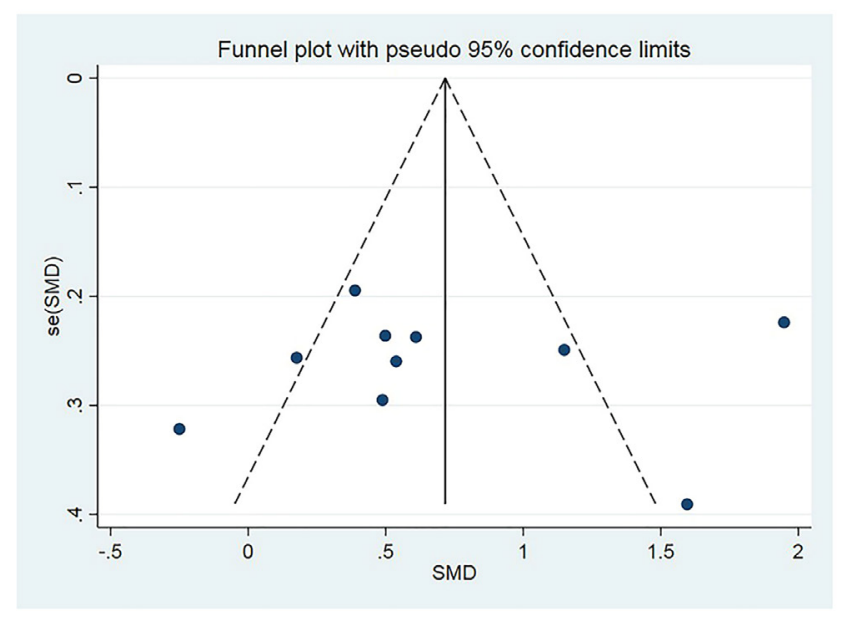

Figure 3. Illustration of funnel plot asymmetry due to heterogeneity between the studies.

\section{DISCUSSION}

\section{Main results}

This study mainly demonstrated an association between EAT and AFACS. Three meta-analyses were carried out to explore the relationship between total EAT volume, LA-specific EAT volume, as well as EAT thickness and AFACS. Their findings were similar, which demonstrated a positive association between EAT and AFACS. Surprisingly, the similar findings between studies that used EAT thickness and EAT volume emphasized the value of echocardiography in the evaluation of EAT because of its security and universality [Ghaderi 2016]. This study, the first effort to clarify the relationship between EAT and AFACS, suggests a new probable imaging indicator of AFACS, which will require further research.

\section{EAT and AFACS}

Although the mechanism underlying the relationship between EAT and AFACS remains unclear, reports demonstrating associations between abundant EAT and AF can be studied. EAT is located between the myocardium and visceral pericardium, primarily over the surfaces of the right ventricle and anterior wall of left ventricle. It also encompasses the atrioventricular grooves and great coronary vessels. It's worth noting that the thickness of EAT over the anterior and lateral walls of the right atrium was the largest [Ansaldo 2019]. Due to its location, it exerts local paracrine effects in the form of increased expression of numerous inflammatory markers; additionally, it modulates the adjacent myocardial tissue with an abundance of ganglionated plexuses of the autonomic nervous system as a metabolically active tissue [Batal 2010; Mazurek 2003; Rossi 2012]. Furthermore, abundant EAT has been demonstrated to be associated with fatty infiltration into the adjacent myocardium using histopathological evidence [Haemers 2017]. Mahajan and Zghaib et al [Mahajan 2015; Zghaib 2016] demonstrated that EAT could cause lower bipolar voltage and electrogram fractionation as electrophysiologic substrates for AF. Besides, there is speculation that the

\begin{tabular}{|c|c|c|c|c|c|c|}
\hline \multicolumn{4}{|c|}{$\begin{array}{l}\text { Meta-regression } \\
\text { REML estimate of between-study variance } \\
\text { \% residual variation due to heterogeneity } \\
\text { Proportion of between-study variance explained } \\
\text { Joint test for all covariates } \\
\text { With Knapp-Hartung modification }\end{array}$} & & $\begin{array}{l}\text { Number of obs } \\
\text { tau2 } \\
\text { I-squared_res } \\
\text { Adj R-squared } \\
\text { Model } F(5,2) \\
\text { Prob }>F\end{array}$ & $\begin{array}{rr}= & 9 \\
= & .2597 \\
= & 72.53 \% \\
= & 35.23 \% \\
= & 1.46 \\
= & 0.4594\end{array}$ \\
\hline _ES & Coef. & Std. Err. & $t$ & $P>|t|$ & [ $95 \%$ Conf. & Interval] \\
\hline Year & -.0441739 & & .0 .33 & & -.626295 & .5379471 \\
\hline Country & -.32856 & & -1.6 & 9 & -1.65 & 1.882334 \\
\hline Number & .0116515 & .0892446 & 1.26 & 0.335 & -.0281246 & .0514277 \\
\hline Methods & -.207898 & .52136 & $-0.4 \theta$ & & -2.451 & 2.035365 \\
\hline Gender & -.0613714 & .038 & -2.84 & 9.178 & -.1998 & .0689888 \\
\hline Quality & .08290 & & & & & .3887274 \\
\hline -cons & 93.019 & 273.8381 & 0.34 & 0.766 & -1085.211 & 1271.249 \\
\hline
\end{tabular}

Figure S1. A meta-regression of heterogeneity

disequilibrium between exceeding oxidative stress and adiponectin's guarding results finally contribute to $\mathrm{AF}$ [Zhou 2020].

AFACS is associated with various factors, including clinical variables, intraoperative surgical variables, electrocardiograph markers, and echocardiographic predictors. In detail, advanced age, hypertension, male sex, right coronary artery stenosis, depressed left ventricular function, remote history of previous AF, combined valve replacement/coronary artery bypass grafting procedures, prolonged aortic cross-clamp and bypass times, PR-interval, QRS duration, abnormal left ventricular systolic and diastolic functions, left ventricular hypertrophy, and increased LA volume may predispose to postoperative AF [Aranki 1996; Hogue 2000; Tran 2015; Zhang 2016; Gu 2017]. AFACS was also demonstrated to be associated with systemic inflammatory response syndrome based on increased serum levels of inflammatory markers, such as interleukin (IL)-6, IL-8, C-reactive protein, tumor necrosis factor- $\alpha$, and indices of neutrophil and platelet activation [Echahidi 2008; Jakubová 2012]. Electrolyte imbalance, especially hypokalemia, also is considered a triggering factor of AFACS [Echahidi 2008; Bharucha 2000].

It was proven that obesity or high $\mathrm{BMI}$ is a risk factor for $\mathrm{AF}$ [Hatem 2015; Wang 2004]. Compared with nonobese people, obese patients have more risk (49\%) of $\mathrm{AF}$, which could be as a result of atrial enlargement, ventricular diastolic dysfunction, as well as increased ectopic fat depot [Wang 2004]. In other reports, EAT was demonstrated to be related to $\mathrm{AF}$ so far as to after regulating with risk factors, such as high BMI [Thanassoulis 2010; Wong 2016]. However, Echahidi et al [Echahidi 2008] reported that metabolic syndrome and obesity were independent risk factors for AFACS. Additionally, EAT was associated with obesity or high BMI [Wong 2016]. Therefore, whether EAT should be regarded as an independent trigger or a modulator of obesity in AFACS requires further clarification.

EAT and AFACs relationship has been confirmed; thus, suggesting that EAT may include the potential to reduce the risk of AFACS. EAT and its accumulation may be reduced by antidiabetic drugs, such as thiazolidinediones [Grosso 2014], glucagon-like peptide 1 receptor agonists [Dutour 2016], dipeptidyl peptidase-4 inhibitors [Lima-Martínez 2016], and sodium-dependent glucose transporter 2 inhibitors [Sato 2018]. Additionally, weight reduction by diet or more sports has been recommended to decrease EAT in the 
Table 2A. Results of sensitivity analysis

\begin{tabular}{lccc}
\hline & Study omitted & Estimate & $\begin{array}{c}95 \% \text { Conf. } \\
\text { Interval }\end{array}$ \\
\hline Drossos (2014) & 0.66642737 & 0.50066859 & 0.8321861 \\
Ozbek (2018) & 0.78323132 & 0.61100805 & 0.95545459 \\
Kogo (total EAT) (2018) & 0.77475089 & 0.60951865 & 0.93998313 \\
Rachwalik (2019) & 0.78021538 & 0.61814988 & 0.94228089 \\
Wang (VHD) (2018) & 0.73446065 & 0.57137752 & 0.89754385 \\
Wang (NVHD) (2018) & 0.67772061 & 0.51735497 & 0.83808631 \\
Liu (2019) & 0.74463749 & 0.57779455 & 0.91148037 \\
Gunturk (2020) & 0.53569818 & 0.36762503 & 0.70377135 \\
Opolski (2015) & 0.72998023 & 0.56324661 & 0.89671385 \\
Kogo (LA-EAT) (2018) & 0.73505941 & 0.57005066 & 0.9000591 \\
Combined & 0.7163113 & 0.5593527 & 0.8732699 \\
\hline
\end{tabular}

EAT, epicardial adipose tissue; VHD, valvular heart disease; NVHD, nonvalvular heart disease; LA, left atrium

interventricular groove, which suggests a correlation with reduced insulin resistance and improved insulin sensitivity indices [Gaborit 2012]. A control study (in vivo and in vitro) that included patients undergoing cardiac surgery for aortic stenosis suggested a robust association between statin therapy and reduced EAT accumulation, which appeared to relate with the anti-inflammatory effects of statins [Parisi 2019]. Considering that EAT contains a large amount of ganglionated plexi, an increasing number of studies aim to lower the inducibility of AF by injecting botulinum toxin into canine epicardial autonomic ganglia [Pokushalov 2015]. Although the idea was hypothetical, it is promising as a potential novel therapeutic option. All these therapies might reduce EAT and decrease the occurrence of several diseases, particularly metabolic diseases, strongly related to AFACS.

Ten studies were categorized into sub-groups, according to the method of assessing EAT, such as echocardiography and CT. Comparatively, CT results were more precise than echocardiography findings because EAT is not evenly distributed around the heart. The optional angle of the echocardiographic probe for two-dimensional imaging may reduce its reproducibility and accuracy; thus, precludes the accurate measurement of pericardial fat or total EAT due to overlap with perivascular adipose tissue. However, in patients with a history of coronary atherosclerosis, which increases EAT thickness, imaging analysis probably provides accurate measurement. Therefore, Nerlekar et al [Nerlekar 2018] demonstrated that EAT measured using CT had poor correlation compared with that measured using echocardiography, and this discrepancy was particularly obvious at greater EAT thickness.

\section{Cause of heterogeneity}

In this study, obvious heterogeneity was observed in two meta-analyses on comparison of EAT volume and thickness in AFACS and non-AFACS patients. The study conducted the
Table 2B. Results of sensitivity analysis leaving out the study by Ertugrul et al.

\begin{tabular}{lccc}
\hline & Study omitted & Estimate & $\begin{array}{c}95 \% \text { Conf. } \\
\text { Interval }\end{array}$ \\
\hline Drossos (2014) & 0.45323172 & 0.27421573 & 0.63224769 \\
Ozbek (2018) & 0.57125425 & 0.38401416 & 0.75849438 \\
Kogo (total EAT) (2018) & 0.58101773 & 0.40266448 & 0.75937104 \\
Rachwalik (2019) & 0.59586072 & 0.42147139 & 0.77025008 \\
Wang (VHD) (2018) & 0.54008275 & 0.71574193 & 0.71574193 \\
Wang (NVHD) (2018) & 0.48201472 & 0.30973798 & 0.65429145 \\
Liu (2019) & 0.54138154 & 0.36099765 & 0.72176534 \\
Opolski (2015) & 0.52456343 & 0.36762503 & 0.70377137 \\
Kogo (LA-EAT)(2018) & 0.53541028 & 0.35734367 & 0.7134769 \\
Combined & 0.5356982 & 0.5593527 & 0.8732699 \\
\hline
\end{tabular}

EAT, epicardial adipose tissue; VHD, valvular heart disease; NVHD, nonvalvular heart disease; LA, left atrium

random effects model in view of the high heterogeneity. However, dividing all studies into three categories-LA-EAT volume, total EAT volume, as well as EAT thickness-reduced the heterogeneity. A meta-regression analysis did not find any possibility of heterogeneity amid different factors (Figure S1), for instance year, country, male sex, EAT evaluation methods, and the quality of studies on account of the STROBE checklist. Furthermore, age, indication of cardiac surgery and EAT measured area, BMI, as well as other classical AF risk factors, were extra probable origins of heterogeneity, which we were unable to evaluate without detailed data. According to the sensitivity analysis (Table 2A), it is suggested that the study by Gunturk et al [Gunturk 2020] could be one of the sources of heterogeneity. (Table 2A) The endpoint of other studies was AF, which was defined according to the European Society of Cardiology guidelines for the management of atrial fibrillation. However, in the study by Ertugrul et al, AFACS was defined as arrhythmic episodes that deteriorate the hemodynamics, last for longer than 20 minutes per episode, or last for longer than 60 minutes during a 24-hour follow-up, which underestimates the incidence of AFACS. If the study by Ertugrul et al is omitted, the sensitivity analysis revealed no heterogeneity (Table $2 \mathrm{~B}$.)

\section{Study limitations}

Due to its observational nature, the current study does not occupy a high position in the pyramid of Evidence-Based Medicine. The studies included were single-center trials with small sample sizes, which might have resulted in unreliable data. The inclusion of only two studies that evaluated LA-EAT thickness is another limitation, because they lacked definitive proof and were unable to arrive at a clear conclusion. Finally, there was a significant degree of heterogeneity between the studies, which may be limiting and was elaborated on in other meta-analyses with $\mathrm{I}^{2}>90 \%$ [Deng 2017; Shimabukuro 2013; Xu 2012]. 


\section{Applications for clinical and future research}

Considering the latest proof, this study emphasized the significant part of EAT in the prediction and potential therapy of AFACS. Given the few previously published studies, future investigations should emphasize: i) well-designed randomized controlled trials with a larger number of participants, or well-designed trials with a synthetical cognitive test at basic line, modulating for baseline and time-varying heterogeneous factors; ii) clarifying the reason and effect association between AF and AFACS; and iii) reporting studies among different ages, genders, and races.

\section{CONCLUSION}

Total EAT volume, LA-EAT volume, and EAT thickness were higher in those with AFACS than non-AFACS. EAT may be a latent indicator and therapeutic target in AFACS. It can be measured either as volume or thickness. Further well-designed prospective research is required to affirm these results to guide the clinical practice.

\section{ACKNOWLEDGEMENT}

This work was supported by the Natural Science Foundation of China (81970282); Biosense Webster Inc. (IIS-324); Shandong Provincial Natural Science Foundation, China, (ZR2018MH002). This was an observational study. The Shandong University Qilu hospital Research Ethics Committee confirmed no ethical approval was required.

\section{REFERENCES}

Ansaldo AM, Montecucco F, Sahebkar A, Dallegri F, Carbone F. 2019. Epicardial adipose tissue and cardiovascular diseases. International Journal of Cardiology. 278:254-260.

Aranki SF, Shaw DP, Adams DH, et al. 1996. Predictors of atrial fibrillation after coronary artery surgery. Current trends and impact on hospital resources. Circulation. 94(3):390-397.

Batal O, Schoenhagen P, Shao M, et al. 2010. Left atrial epicardial adiposity and atrial fibrillation. Circulation Arrhythmia and Electrophysiology. 3(3):230-236.

Bharucha DB, Kowey PR. 2000. Management and prevention of atrial fibrillation after cardiovascular surgery. The American journal of cardiology. 85(10a):20d-24d.

Cheng VY, Dey D, Tamarappoo B, et al. 2010. Pericardial fat burden on ECG-gated noncontrast CT in asymptomatic patients who subsequently experience adverse cardiovascular events. JACC Cardiovascular Imaging. 3(4):352-360

D'Agostino RS, Jacobs JP, Badhwar V, et al. 2016. The Society of Thoracic Surgeons Adult Cardiac Surgery Database: 2016 Update on Outcomes and Quality. The Annals of Thoracic Surgery. 101(1):24-32.

D'Agostino RS, Jacobs JP, Badhwar V, et al. 2018. The Society of Thoracic Surgeons Adult Cardiac Surgery Database: 2018 Update on Outcomes and Quality. The Annals of Thoracic Surgery. 105(1):15-23.

Deng H, Bai Y, Shantsila A, Fauchier L, Potpara TS, Lip GYH. 2017.
Clinical scores for outcomes of rhythm control or arrhythmia progression in patients with atrial fibrillation: a systematic review. Clinical research in cardiology: official journal of the German Cardiac Society. 106(10):813-823.

Ding J, Hsu FC, Harris TB, et al. 2009. The association of pericardial fat with incident coronary heart disease: the Multi-Ethnic Study of Atherosclerosis (MESA). The American Journal of Clinical Nutrition. 90(3):499-504.

Drossos G, Koutsogiannidis CP, Ananiadou O, et al. 2014. Pericardial fat is strongly associated with atrial fibrillation after coronary artery bypass graft surgery†. European journal of cardiothoracic surgery: official journal of the European Association for Cardiothoracic Surgery. 46(6):10141020; discussion 1020 .

Dutour A, Abdesselam I, Ancel P, et al. 2016. Exenatide decreases liver fat content and epicardial adipose tissue in patients with obesity and type 2 diabetes: a prospective randomized clinical trial using magnetic resonance imaging and spectroscopy. Diabetes, Obesity \& Metabolism. 18(9):882-891.

Echahidi N, Pibarot P, O'Hara G, Mathieu P. 2008. Mechanisms, prevention, and treatment of atrial fibrillation after cardiac surgery. Journal of the American College of Cardiology. 51(8):793-801.

Gaborit B, Jacquier A, Kober F, et al. 2012. Effects of bariatric surgery on cardiac ectopic fat: lesser decrease in epicardial fat compared to visceral fat loss and no change in myocardial triglyceride content. Journal of the American College of Cardiology. 60(15):1381-1389.

Gaita F, Scaglione M, Battaglia A, et al. 2018. Very long-term outcome following transcatheter ablation of atrial fibrillation. Are results maintained after 10 years of follow up? Europace: European pacing, arrhythmias, and cardiac electrophysiology: journal of the working groups on cardiac pacing, arrhythmias, and cardiac cellular electrophysiology of the European Society of Cardiology. 20(3):443-450.

Ghaderi F, Eshraghi A, Shamloo AS, Mousavi S. 2016. Association of Epicardial and Pericardial Fat Thickness with Coronary Artery Disease. Electronic physician. 8(9):2982-2989.

Grosso AF, de Oliveira SF, Higuchi Mde L, Favarato D, Dallan LA, da Luz PL. 2014. Synergistic anti-inflammatory effect: simvastatin and pioglitazone reduce inflammatory markers of plasma and epicardial adipose tissue of coronary patients with metabolic syndrome. Diabetology \& Metabolic Syndrome. 6(1):47.

Gu J, Andreasen JJ, Melgaard J, et al. 2017. Preoperative Electrocardiogram Score for Predicting New-Onset Postoperative Atrial Fibrillation in Patients Undergoing Cardiac Surgery. Journal of Cardiothoracic and Vascular Anesthesia. 31(1):69-76.

Gunturk EE, Topuz M, Serhatlioğlu F, Akkaya H. 2020. Echocardiographically Measured Epicardial Fat Predicts New-onset Atrial Fibrillation after Cardiac Surgery. Brazilian Journal of Cardiovascular Surgery. 35(3):339-345.

Haemers P, Hamdi H, Guedj K, et al. 2017. Atrial fibrillation is associated with the fibrotic remodelling of adipose tissue in the subepicardium of human and sheep atria. European Heart Journal. 38(1):53-61.

Hatem SN. 2015. Atrial Fibrillation and Obesity: Not Just a Coincidence. Journal of the American College of Cardiology. 2015;66(1):12-13.

Hogue CW, Jr., Hyder ML. 2000. Atrial fibrillation after cardiac operation: risks, mechanisms, and treatment. The Annals of Thoracic Surgery. 69(1):300-306.

Jakubová M, Mitro P, Stančák B, et al. 2012. The occurrence of postoperative atrial fibrillation according to different surgical settings in 
cardiac surgery patients. Interactive Cardiovascular and Thoracic Surgery. 15(6): 1007-1012.

Kogo H, Sezai A, Osaka S, Shiono M, Tanaka M. 2019. Does Epicardial Adipose Tissue Influence Postoperative Atrial Fibrillation? Annals of thoracic and cardiovascular surgery: official journal of the Association of Thoracic and Cardiovascular Surgeons of Asia. 25(3):149-157.

Lima-Martínez MM, Paoli M, Rodney M, et al. 2016. Effect of sitagliptin on epicardial fat thickness in subjects with type 2 diabetes and obesity: a pilot study. Endocrine. 51(3):448-455.

Liu Q, Zhang F, Yang M, ZhongJ. 2020. Increasing Level of Interleukin-1 in Epicardial Adipose Tissue Is Associated with Persistent Atrial Fibrillation. Journal of interferon \& cytokine research: the official journal of the International Society for Interferon and Cytokine Research. 40(1):64-69.

Mahabadi AA, Massaro JM, Rosito GA, et al. 2009. Association of pericardial fat, intrathoracic fat, and visceral abdominal fat with cardiovascular disease burden: the Framingham Heart Study. European Heart Journal. 30(7):850-856.

Mahajan R, Lau DH, Brooks AG, et al. 2015. Electrophysiological, Electroanatomical, and Structural Remodeling of the Atria as Consequences of Sustained Obesity. Journal of the American College of Cardiology. 66(1):1-11.

Mazurek T, Zhang L, Zalewski A, et al. 2003. Human epicardial adipose tissue is a source of inflammatory mediators. Circulation. 108(20):2460-2466.

Nerlekar N, Baey YW, Brown AJ, et al. 2018. Poor Correlation, Reproducibility, and Agreement Between Volumetric Versus Linear Epicardial Adipose Tissue Measurement: A 3D Computed Tomography Versus 2D Echocardiography Comparison. JACC Cardiovascular Imaging. 11(7):1035-1036.

Opolski MP, Staruch AD, Kusmierczyk M, et al. 2015. Computed tomography angiography for prediction of atrial fibrillation after coronary artery bypass grafting: proof of concept. Journal of Cardiology. 65(4):285-292.

Ozbek K, Katlandur H, Arik B, et al. 2018. Role of red blood cell distribution width and epicardial fat in atrial fibrillation after cardiopulmonary bypass. Electron J Gen Med. 15(2):7.

Parisi V, Petraglia L, D'Esposito V, et al. 2019. Statin therapy modulates thickness and inflammatory profile of human epicardial adipose tissue. International Journal of Cardiology. 274:326-330.

Pokushalov E, Kozlov B, Romanov A, et al. 2015. Long-Term Suppression of Atrial Fibrillation by Botulinum Toxin Injection Into Epicardial Fat Pads in Patients Undergoing Cardiac Surgery: One-Year Follow-Up of a Randomized Pilot Study. Circulation Arrhythmia and Electrophysiology. 8(6):1334-1341.

Rachwalik M, Obremska M, Zyśko D, Matusiewicz M, Ściborski K, Jasiński M. 2019. The concentration of resistin in perivascular adipose tissue after CABG and postoperative atrial fibrillation. BMC Cardiovascular Disorders. 19(1):294.

Rosito GA, Massaro JM, Hoffmann U, et al. 2008. Pericardial fat, visceral abdominal fat, cardiovascular disease risk factors, and vascular calcification in a community-based sample: the Framingham Heart Study. Circulation. 117(5):605-613.

Rossi P, Ricci A, De Paulis R, et al. 2012. Epicardial ganglionated plexus stimulation decreases postoperative inflammatory response in humans. Heart Rhythm. 9(6):943-950.

Sato T, Aizawa Y, Yuasa S, et al. 2018. The effect of dapagliflozin treatment on epicardial adipose tissue volume. Cardiovascular Diabetology. 17(1):6.

Schwann TA, Al-Shaar L, Engoren MC, et al. 2018. Effect of new-onset atrial fibrillation on cause-specific late mortality after coronary artery bypass grafting surgery. European journal of cardio-thoracic surgery: Official Journal of the European Association for Cardio-thoracic Surgery. 54(2):294-301.

Shen J, Lall S, Zheng V, Buckley P, Damiano RJ, Jr., Schuessler RB. 2011. The persistent problem of new-onset postoperative atrial fibrillation: a single-institution experience over two decades. The Journal of Thoracic and Cardiovascular Surgery. 141(2):559-570.

Shimabukuro M, Hirata Y, Tabata M, et al. 2013. Epicardial adipose tissue volume and adipocytokine imbalance are strongly linked to human coronary atherosclerosis. Arteriosclerosis, Thrombosis, and Vascular Biology. 33(5):1077-1084.

Thanassoulis G, Massaro JM, O'Donnell CJ, et al. 2010. Pericardial fat is associated with prevalent atrial fibrillation: the Framingham Heart Study. Circulation Arrhythmia and Electrophysiology. 3(4):345-350.

Tran DT, Perry JJ, Dupuis JY, Elmestekawy E, Wells GA. 2015. Predicting New-Onset Postoperative Atrial Fibrillation in Cardiac Surgery Patients. Journal of Cardiothoracic and Vascular Anesthesia. 29(5):1117-1126.

Venteclef N, Guglielmi V, Balse E, et al. 2015. Human epicardial adipose tissue induces fibrosis of the atrial myocardium through the secretion of adipo-fibrokines. European Heart Journal. 36(13):795-805a.

von Elm E, Altman DG, Egger M, Pocock SJ, Gøtzsche PC, Vandenbroucke JP. 2014. The Strengthening the Reporting of Observational Studies in Epidemiology (STROBE) Statement: guidelines for reporting observational studies. International Journal of Surgery (London, England). 12(12):1495-1499.

Wang TJ, Parise H, Levy D, et al. 2004. Obesity and the risk of newonset atrial fibrillation. Jama. 292(20):2471-2477.

Wang Q, Min J, Jia L, et al. 2019. Human Epicardial Adipose Tissue Activin A Expression Predicts Occurrence of Postoperative Atrial Fibrillation in Patients Receiving Cardiac Surgery. Heart, Lung \& Circulation. 28(11):1697-1705.

Wong CX, Abed HS, Molaee P, et al. 2011. Pericardial fat is associated with atrial fibrillation severity and ablation outcome. Journal of the American College of Cardiology. 57(17):1745-1751.

Wong CX, Ganesan AN, Selvanayagam JB. 2017. Epicardial fat and atrial fibrillation: current evidence, potential mechanisms, clinical implications, and future directions. European Heart Journal. 38(17):1294-1302.

Wong CX, Sun MT, Odutayo A, et al. 2016. Associations of Epicardial, Abdominal, and Overall Adiposity With Atrial Fibrillation. Circulation Arrhythmia and Electrophysiology. 9(12).

Xu Y, Cheng X, Hong K, Huang C, Wan L. 2012. How to interpret epicardial adipose tissue as a cause of coronary artery disease: a metaanalysis. Coronary Artery Disease. 23(4):227-233.

Zghaib T, Ipek EG, Zahid S, et al. 2016. Association of left atrial epicardial adipose tissue with electrogram bipolar voltage and fractionation: Electrophysiologic substrates for atrial fibrillation. Heart Rhythm. 13(12):2333-2339.

Zhang W, Liu W, Chew ST, Shen L, Ti LK. 2016. A Clinical Prediction Model for Postcardiac Surgery Atrial Fibrillation in an Asian Population. Anesthesia and Analgesia. 123(2):283-289.

Zhou M, Wang H, Chen J, Zhao L. 2020. Epicardial adipose tissue and atrial fibrillation: Possible mechanisms, potential therapies, and future directions. Pacing and Clinical Electrophysiology: PACE. 43(1):133-145. 